\title{
AGROMETEOROLOGIA
}

Nota

\section{TENDÊNCIAS E VARIAÇÕES CLIMÁTICAS EM SÉRIES ANUAIS DE PRECIPITAÇÃO PLUVIAL DO ESTADO DE SÃO PAULO (1)}

\author{
GABRIEL CONSTANTINO BLAIN $\left({ }^{2 *}\right)$
}

\begin{abstract}
RESUMO
O objetivo do trabalho foi detectar tendências e variações climáticas em oito séries de totais anuais de precipitação pluvial do Estado de São Paulo. A fim de obter melhor adequação entre as probabilidades dos erros tipo I e II, as análises foram realizadas do ponto de vista paramétrico e não paramétrico. Nas localidades de Jundiaí e Pindorama foram detectadas marcantes elevações no regime anual dos totais de precipitação. A mesma característica foi observada na localidade de Monte Alegre do Sul, embora com menor significância do que nessas duas primeiras séries. Nas localidades de Campinas, Cordeirópolis, Mococa e Ribeirão Preto não houve indícios de alterações climáticas. Na localidade de Ubatuba foi detectada queda no regime dessa variável meteorológica.
\end{abstract}

Palavras-chave: Mudança climática, paramétrico, não paramétrico.

\author{
ABSTRACT \\ CLIMATE TRENDS AND VARIATIONS RELATED TO TOTAL ANNUAL \\ PRECIPITATION SERIES OF THE STATE OF SÃO PAULO, BRAZIL
}

The aim of the work was to detect trends and climate variations in eight annual precipitation series of the State of São Paulo, Brazil. The investigations was carried out under parametric and non parametric methods in order to obtain a better balance between probability type I and type II errors. Jundiaí and Pindorama presented significant increasing trends in the annual precipitation amounts. In the series of Monte Alegre do Sul those increasing trends had a lower degree of significance. No significant climate change was detected in the series of Campinas, Cordeirópolis, Ribeirão Preto and, Mococa. A decreased trend was observed in the annual precipitation series of Ubatuba.

Key words: Climate change, parametric, non parametric.

(1) Recebido para publicação em 27 de março de 2009 e aceito em 15 de dezembro de 2009.

$\left(^{2}\right)$ Instituto Agronômico de Campinas (IAC/APTA/SAA), Caixa Postal 28, 13001-970 Campinas (SP). E-mail: gabriel@iac.sp.gov.br

$\left({ }^{*}\right)$ Autor correspondente. 
Atualmente, uma das maiores preocupações da comunidade científica mundial tem sido o tema mudança climática. Segundo o relatório do IPCC (2007), esse termo refere-se a alterações nos padrões climáticos, que podem ser identificadas por meio de variações persistentes por longos períodos, da ordem de décadas, na média aritmética e/ou em outras medidas da variabilidade climática, independentes de suas causas. Entretanto, é importante ressaltar que estudos sobre alterações nos padrões climáticos globais não são recentes. Na nota técnica n. ${ }^{\circ} 79$ (Омм, 1966), por exemplo, afirma-se que a análise de séries temporais meteorológicas demonstra claramente a inconstância do clima. Para esse relatório, mudança climática é um termo genérico que engloba todas as formas de inconstância nos padrões climáticos, tais como tendências ou variações, independentemente de suas causas. Esses últimos dois conceitos são definidos por:

Tendência climática: caracterizada por uma elevação ou diminuição suave (smooth) e monótona nos valores médios de uma série meteorológica. Não é, necessariamente, restrita a tendências lineares em função do tempo, mas deve conter apenas um máximo ou mínimo no ponto final da série. Variações climáticas: flutuação ou componente desta em escala de tempo suficientemente longa, capaz de resultar em inconstância nos parâmetros estatísticos relativos a períodos sucessivos de pelo menos 30 anos da variável meteorológica em questão.

Alexander et al. (2006), Haylock et al. (2006), MARENGO et al. (2007) e VINCENT et al. (2005) apresentam exemplos de análises estatísticas de alterações nos padrões climáticos globais com base em séries temporais meteorológicas. Sob o ponto de vista matemático, essas investigações podem ser desenvolvidas por métodos paramétricos e não paramétricos. A justificativa em adotar essas duas formas de estudo é baseada em afirmações como as de DALE (1968) e SANSIGOlo e NerY (2000), relativas ao maior embasamento estatístico da descrição climática quando são utilizados modelos teóricos. Com isso, pode-se inferir que o primeiro passo no estudo de possíveis variações climáticas, é o ajuste das séries meteorológicas a uma distribuição paramétrica. A análise de possíveis mudanças climáticas é realizada por meio do estudo dos parâmetros das distribuições relativos a duas ou mais amostras da série original, diminuindo, com isso, a probabilidade de que as diferenças entre esses subperíodos sejam atribuídas a deficiências no tamanho da amostra ou, em outras palavras, a falhas na descrição climática da região.

Em contrapartida, é também interessante ressaltar FREI e SCHAR (2000) quando afirmam que análises de tendências em longas séries meteorológicas confrontam-se com a dificuldade fundamental de que as variações estocásticas limitam a acurácia com a qual possíveis alterações possam ser detectadas. Esses autores especificam os dois tipos de erro que podem ocorrer: a) indicação de tendências climáticas, quando há apenas flutuações essencialmente controladas pelas variações estocásticas (erro tipo I); b) uma real tendência climática não é identificada por estar "encoberta" por flutuações estocásticas de pequenas escalas temporais (erro tipo II). No presente caso, apesar do "amaciamento" resultante do ajuste das distribuições empíricas a uma distribuição teórica diminuir a probabilidade do erro tipo I aumenta, obviamente, a do tipo II. Com isso, torna-se também interessante analisar possíveis variações e tendências climáticas considerando testes não paramétricos.

O objetivo do trabalho foi detectar tendências e variações climáticas em oito séries de totais anuais de precipitação pluvial (PRE) do Estado de São Paulo.

Foram utilizados dados anuais de precipitação pluvial de oito localidades pertencentes à Secretaria de Agricultura e Abastecimento do Estado de São Paulo (SAA). Na figura 1, são ilustradas as coordenadas geográficas dos postos utilizados. Exceto Pindorama (1951 a 2007), todas as demais localidades da SAA foram escolhidas de forma que gerassem duas amostras de 30 anos de dados (1948 a 2007).

Os métodos estatísticos utilizados foram: teste t-student, teste $F$, teste da razão da verossimilhança $\left(\Lambda^{*}\right)$ e teste de Mann-Kendall (MK). Conceitualmente, os três primeiros são denominados de paramétricos por assumirem que a amostra pode ser ajustada a uma distribuição teórica. O MK não faz distinção quanto à distribuição que os dados são oriundos, sendo, com isso, considerado não-paramétrico. Descrições detalhadas das propriedades e limitações dos testes t e F podem ser constatadas, entre outros, em PANOFSKY e BRIER (1968), WiLks $(1995,2006)$ e Von Storch e Zwiers (1999).

Os testes de aderência Kolmogorav-Smirnov (KS) e qui-quadrado $\left(\chi^{2}\right)$, descritos em diversos trabalhos tais como PANOFsky e Brier (1968), Von Storch e Zwiers (1999) e WiLKS (2006) foram utilizados para verificar os ajustes das séries e das amostras de PRE à distribuição normal $(\mathrm{DN})$.

$\mathrm{O} \Lambda^{*}$ é indicado por Schickedanz e KRAuSE (1969) e Wilks (2006) como interessante alternativa para verificar se distintos períodos de uma série histórica podem ser considerados oriundos da mesma população com distribuição paramétrica conhecida. No presente caso, sob $\mathrm{H}_{0^{\prime}}$ a distribuição dos valores de $\Lambda^{*}$ é qui-quadrado com 2 graus de liberdade. Ho considera que os distintos subperíodos analisados pertencem à "mesma distribuição", rejeitada quando a verossimilhança associada a $\mathrm{H}_{\mathrm{A}^{\prime}}$, aqui definida como Ho não é verdadeira, sendo suficientemente elevada 


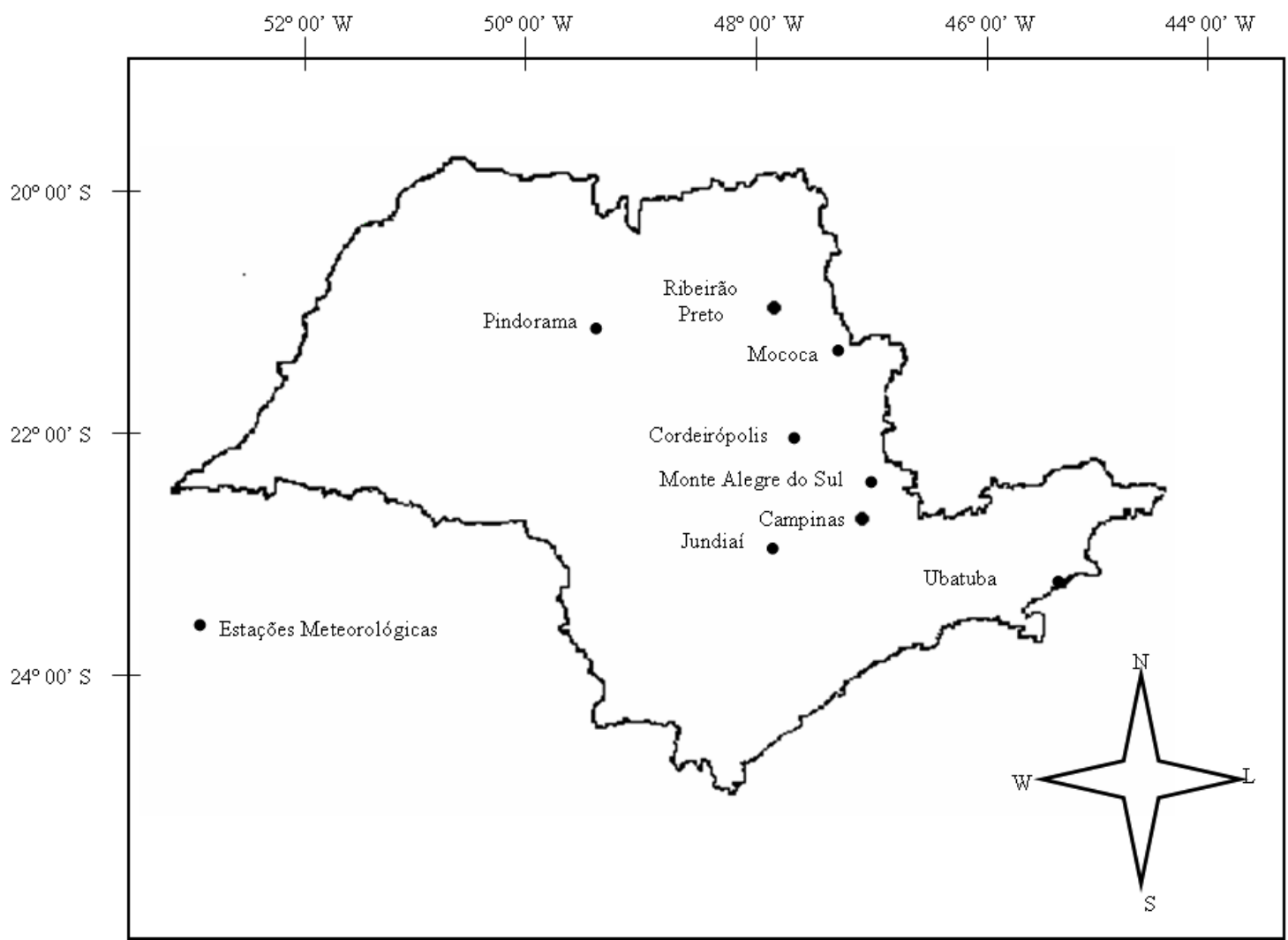

Figura 1. Postos meteorológicos pertencentes à Secretaria de Agricultura e Abastecimento do Estado de São Paulo.

para que as diferenças estatísticas entre os subperíodos sejam consideradas apenas variações amostrais. Para o nível 10\% de significância, o valor crítico é 4,61. O teste é definido da seguinte forma:

$$
\Lambda^{*}=2 \operatorname{Ln}\left[\Lambda\left(\mathrm{H}_{\mathrm{A}}\right) / \Lambda(\mathrm{Ho})\right]=2\left[\mathrm{~L}\left(\mathrm{H}_{\mathrm{A}}\right)-\mathrm{L}(\mathrm{Ho})\right]
$$

Adotando as duas amostras relativas aos anos de 1948/1977 e 1978/ 2007 compostas por $x_{\mathrm{i}}$ valores, o teste $\Lambda^{*}$, quando utilizada a DN, é calculado por:

$\Lambda^{*}=2\left\{\left[\sum_{i=1948}^{2007} L_{1}\left(\mu_{1}, \sigma_{1} ; x_{i}\right)+\sum_{i=1948}^{1977} L_{2}\left(\mu_{2}, \sigma_{2} ; x_{i}\right)\right]-\sum_{i=1948}^{200} L\left(\mu, \sigma ; x_{i}\right)\right\}$

$\mathrm{L}\left(\mu, \sigma ; \mathrm{x}_{\mathrm{i}}\right)=-\ln (\sigma)-\ln \left[(2 \pi)^{1 / 2}\right]-(1 / \sigma)\left\{\sum\left[\left(\mathrm{x}_{\mathrm{i}}-\mu\right)^{2}\right]\right\}$

$\sigma$ e $\mu$ são, respectivamente, o desvio-padrão e a média aritmética dos dados.

Analisando a equação 1.2, verifica-se que o fator L é simplesmente o logaritmo natural da função densidade de probabilidade, para um dado $x_{i}$, associada à distribuição teórica para a qual a amostra foi ajustada.
Proposto por ManN (1945) e Kendall (1967) o teste não paramétrico de Mann-Kendall (MK) pode ser definido, para uma série $Z=\left\{Z_{1^{\prime}} Z_{2^{\prime}}, \ldots Z_{i^{\prime}} Z_{j^{\prime}} \ldots . . Z_{n}\right)$ por:

$\mathrm{T}=\sum_{\mathrm{j}<1} \operatorname{sinal}\left(\mathrm{Z}_{\mathrm{i}}-\mathrm{Z}_{\mathrm{j}}\right)$

para:

$\operatorname{sinal}\left(Z_{\mathrm{i}}-Z_{\mathrm{j}}\right)=\left\{\begin{array}{l}1 ; \text { para } \mathrm{Z}_{\mathrm{i}}-\mathrm{Z}_{\mathrm{j}}>0 \\ 0 ; \text { para } \mathrm{Z}_{\mathrm{i}}-\mathrm{Z}_{\mathrm{j}}=0 \\ -1 ; \text { para } \mathrm{Z}_{\mathrm{i}}-\mathrm{Z}_{\mathrm{j}}<0\end{array}\right\}$

Quando não há presença de tendências (Ho), T é normalmente distribuído com média $\mathrm{E}(\mathrm{T})=0$ e variância $\operatorname{Var}(\mathrm{T})$ estimado por:

$\mathrm{MK}=\left\{\begin{array}{l}\frac{\mathrm{T}-1}{\sqrt{\operatorname{Var}(\mathrm{T})}} ; \text { para } \mathrm{T}>0 \\ 0 ; \text { para } \mathrm{T}=0 \\ \frac{\mathrm{T}+1}{\sqrt{\operatorname{Var}(\mathrm{T})}} ; \text { para } \mathrm{T}<0\end{array}\right\}$

para 

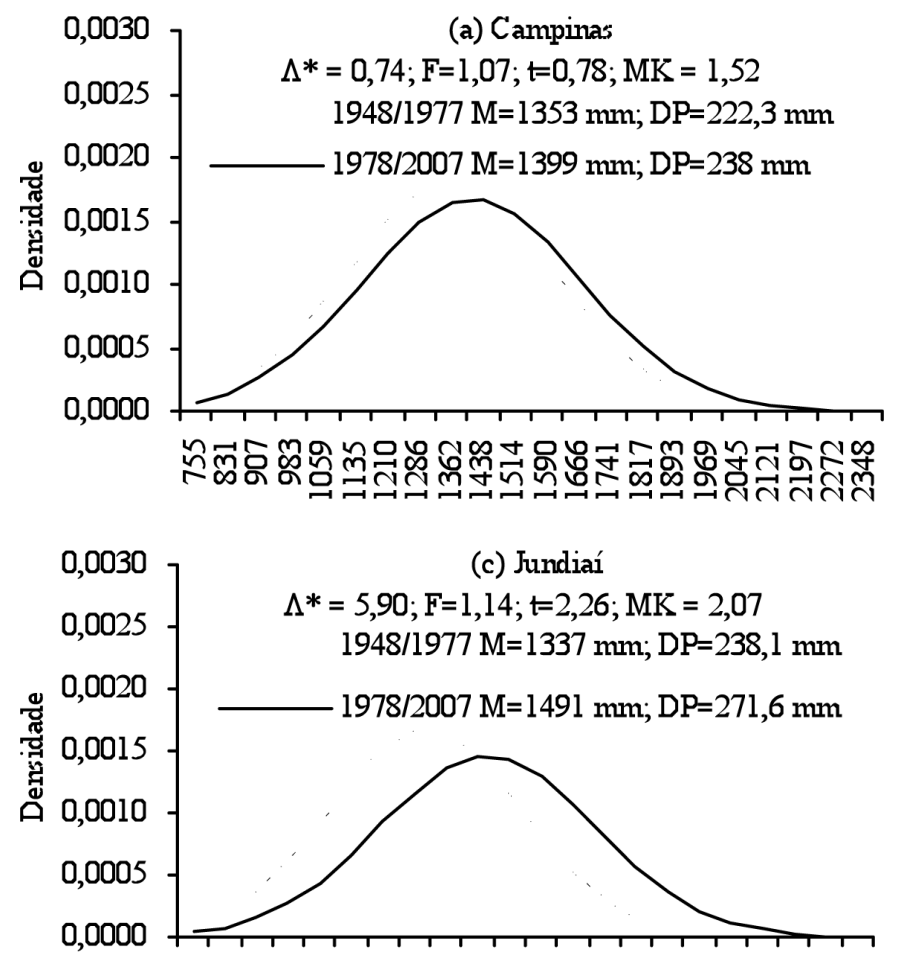

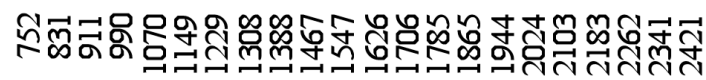
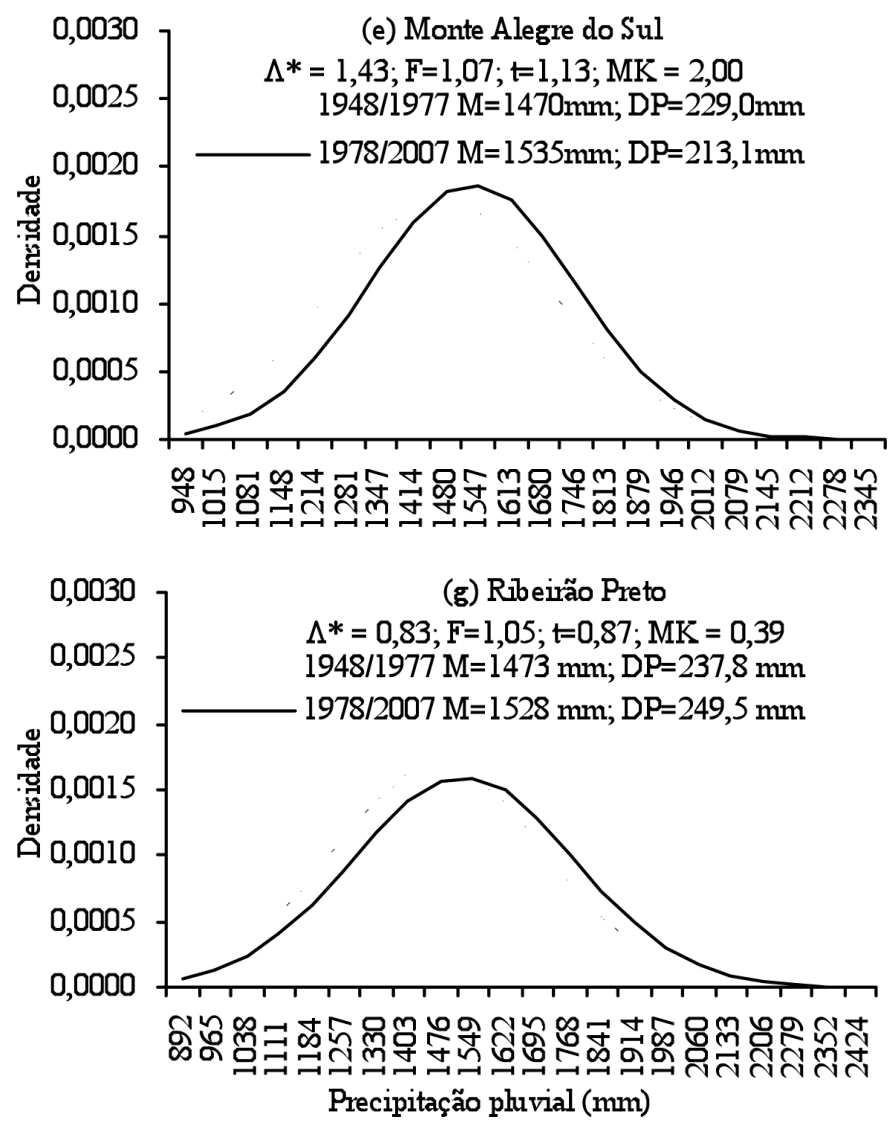
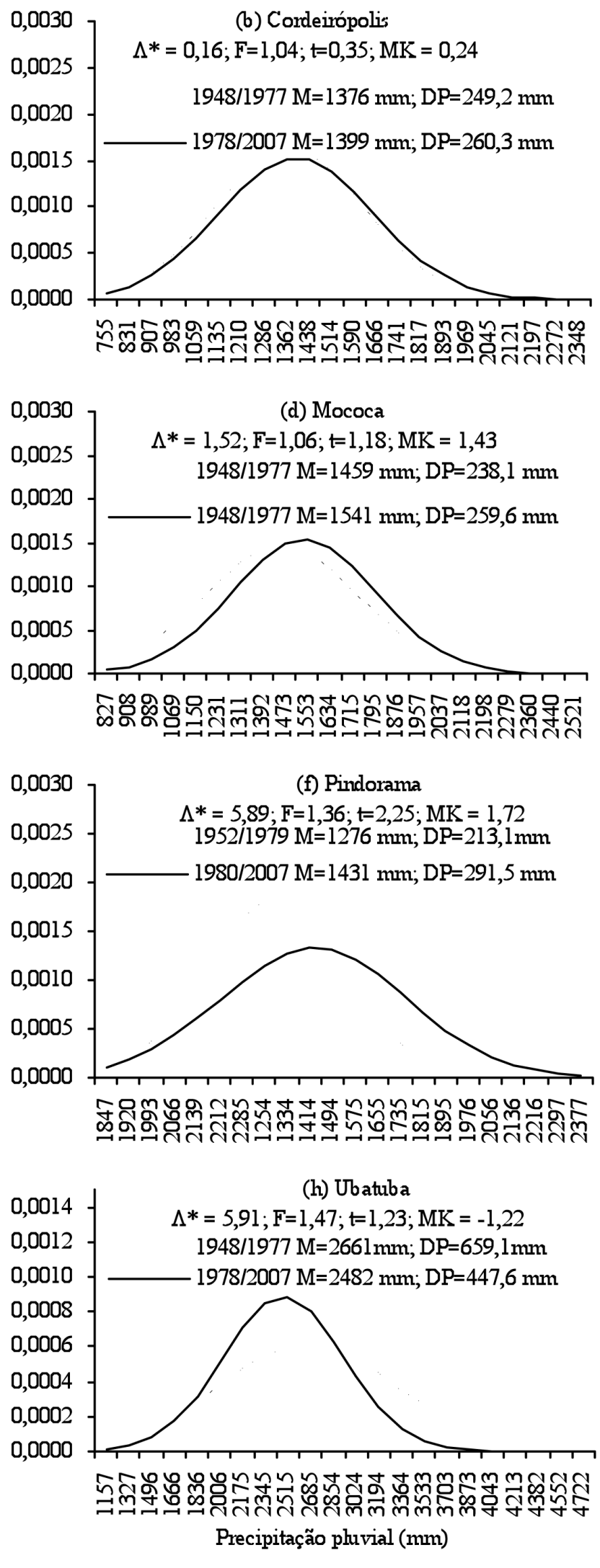

Figura 2. Aplicação dos testes razão da verossimilhança $\left(\Lambda^{*}\right), \mathrm{F}, \mathrm{t}, \mathrm{Mann}-\mathrm{Kendall}(\mathrm{MK})$ para as localidades do estudo. Os respectivos valores críticos são 4,61; 1,86 (1,90 Pindorama); 2,01 e [-1,96 a +1,96]. A média aritmética (M) e o desvio-padrão (DP) de cada subperíodo também são ilustrados. 
$\operatorname{Var}(T)=\frac{n(n-1)(2 n+5)}{18}$

Autores como MARENGO et al. (2007) utilizam o nível de significância $\mathrm{a}=0,05$ para a aplicação desse método. Adotando esse mesmo patamar, Ho não é rejeitada sempre que $-1,96<\mathrm{MK}<1,96$. Tendências de elevação resultam em valores positivos do $\mathrm{MK}$, ao passo que valores negativos deste teste indicam tendência de queda nos dados da série sob análise.

Comparando os testes $\Lambda^{*}$, t e F, nota-se que esses dois últimos apenas analisam as medidas de posição estatística média e variância amostral respectivamente. $\mathrm{O} \Lambda^{*}$, em contrapartida, investiga a probabilidade teórica de ocorrência associada a cada valor $x_{i}$ oriundo de cada período amostral. Nesse contexto, analisando as equações 1 a 1.2 , observa-se que o $\Lambda^{*}$ é bastante adequado para verificar variações nos parâmetros das funções densidade de probabilidade associadas às diferentes amostras de uma mesma série. Contudo, o sequenciamento temporal dos valores constituintes desses subperíodos não é analisado.

Por meio das equações 2 e 3, verifica-se que essa disposição temporal tem profunda influência no valor final do MK. Assim, observa-se que esse método não paramétrico, é fundamentalmente desenvolvido para a detecção de tendências monótonas em uma série temporal. Do ponto de vista meteorológico, essa diferença entre o $\Lambda^{*}$ e o $\mathrm{MK}$ pode ser interpretada considerando as definições de variações e tendências climáticas descritas no item 1. Pode-se, portanto, inferir que o $\Lambda^{*}$ é mais adequado para detecção de variações, ao passo que o MK é voltado a detecção de tendências climáticas. Os testes $\mathrm{KS}$ e $\chi^{2}$ comprovam o ajuste das séries de PRE a DN, permitindo a aplicação dos testes $\Lambda^{*}, \mathrm{~F}$ e t, conforme ilustrado na figura 2 .

Na figura 2, observa-se que as séries de PRE anual de Campinas, Cordeirópolis, Mococa e Ribeirão Preto, não possuem indícios significativos de variações ou tendências climáticas. Essa afirmação é baseada no fato de que nenhum dos métodos empregados, paramétricos e não paramétricos, proporcionou valores fora dos respectivos limites de rejeição de Ho.

Na localidade de Monte Alegre do Sul, apenas o MK indicou tendências significativas de elevação monótona nos valores anuais de PRE. Contudo, essa tendência não foi capaz de alterar significativamente as probabilidades teóricas de ocorrência dos valores anuais desse elemento meteorológico associadas às distintas amostras, conforme indicado pelo valor não significativo do teste $\Lambda^{*}$. Na série de Jundiaí são detectados os indícios mais marcantes de tendências de elevação e variação de ordem climática nos valores de
PRE, entre todas as localidades analisadas. A exceção do teste $\mathrm{F}$, todos os métodos aplicados representaram valores fora dos limites críticos de rejeição de Ho. Nesse contexto, analisando as formas gráficas das funções densidade associadas às DN amostrais, inferese que a significativa alteração nas probabilidades paramétricas de ocorrência dos totais de PRE está fundamentalmente relacionada à tendência monótona de elevação, detectada pelo MK. Essa afirmação também é corroborada pela homocedasticidade das variâncias amostrais. A localidade de Ubatuba foi a única a ter valores negativos, embora não significativo, do MK. Verifica-se também que o significativo valor do teste $\Lambda^{*}$ está associado ao deslocamento à esquerda e ao aumento na probabilidade de ocorrência dos valores próximos à média aritmética da amostra relativa de 1978 a 2007.

De forma geral, não há marcantes alterações no regime anual de PRE das localidades de São Paulo. Contudo, à exceção da localidade litorânea de Ubatuba, foram observadas tendências pouco significativas de elevação no regime dessa variável meteorológica, tendo em vista o deslocamento à direita das funções densidade de probabilidade, especialmente na série de Jundiaí, associadas aos últimos 30 anos.

Nas localidades de Jundiaí e Pindorama foram detectados marcantes indícios de alterações de ordem climática no regime anual dos totais de precipitação pluvial nos diferentes períodos analisados. Verifica-se que essas alterações estão fundamentalmente ligadas à elevação do regime dessa variável meteorológica nos últimos 30 anos.

Na localidade de Monte Alegre do Sul, verificase tendência pouco significativa de elevação nos totais anuais de precipitação pluvial ocorrida nos últimos anos dessa série. Nas localidades de Campinas, Cordeirópolis, Mococa e Ribeirão Preto não houve indícios de alterações climáticas para essa variável meteorológica. Na localidade de Ubatuba foram observados indícios de queda no regime de precipitação pluvial nos últimos 30 anos analisados.

\section{REFERÊNCIAS}

ALEXANDER, L.V.; ZHANG, X.; PETERSON, T.C.; CAESAR, J.; GLEASON, B.; TANK, A.M.G; HAYLOCK, M.; COLLINS, D.; TREVIN, B.; RAHIMZADEH, F.; TAGIPOU, A.; RUPA KUMAR, K.; REVADEKAR, J.; GRIFFITHS, G.; VINCENT, L.; STEPHENSON, D.; BURN, J.; AGUILLAR, E.; TAYLOR, M.; NEW, M.; ZHAI, P.; RUSTICUCCI, M.; VASQUEZ-AGUIRRE, J.L. Global observed changes in daily climate extremes of temperature and precipitation. Journal of Geophysical Research, v.111, p.1-22, 2006.

DALE, R.F. Applied Climatogy. 1.ed. Indiana: Purdue University, 1968. 125p. 
FREI, C.; SCHAR, C. Detection probability of trends in rare events: Theory and application to heavy precipitation in the Alpine region. Journal of Climate, v.14, p.1568-1584, 2000.

HAYLOCK, M.R.; PETERSON, T.C.; ALVES, L.M.; AMBRIZZI, T.;ANUNCIAÇÃO, Y.M.T.; BAEZ, J.; BARROS, V.R.;BERLATO, M.A.; BIDEGAIN, M.; CORONEL, G.; CORRADI, V.; GARCIA, V.J.; GRIMM, A.M.; KAROLY, D.; MARENGO, J.A.; MARINO, M.B.; MONCUNILL, D.F.; NECHET, D.; QUINTANA, J.; REBELLO, E.; RUSTICUCCI, M.; SANTOS, J.L.; TREBEJO, I.; VINCENT L.A. Trends in total and extreme South American rainfall in 1960-2000 and links with sea surface temperature. Journal of Climate, v.19, p.1490-1512, 2006.

IPCC, Climate Change 2007: The Physical Science Basis, Contribution of Working Group I to the Fourth Assessment Report of the Intergovernmental Panel on Climate Change, HOUGHTON, J.T. (Ed.). In: Cambridge University Press, Cambridge: 2007.

KENDALL, M.A.; STUART, A. The advanced theory of statistics. 1.ed. Londres: Charles Griffin, 1967. 690p.

MANN, H.B. Non-parametric tests against trend. Econometrica, v.13, p.245-259, 1945.

MARENGO, J.; NOBRE, C.; RAIGOZA, D.; VALVERDE, M.; PISNITCHENKO, I.A.; OLIVEIRA, J.C.M. Boletim do Projeto: uso de cenários de mudanças climáticas regionais em estudos de vulnerabilidade e adaptação no Brasil e na América do Sul (GOF-UK-CPTEC), 2007. Disponível em: <www.cptec.inpe. br/mudancas_climaticas $>$. Acesso em: 1. ${ }^{\circ}$ fev. 2009.

ORGANIZAÇÃO METEOROLÓGICA MUNDIAL. Climatic Change. 1.ed. Geneva: WMO, 1966. 80p.
PANOFSKY, H.A.; BRIERG, W. Some applications of statistics to meteorology. 1. ed. Florida: University Park, 1968. 224p.

SANSIGOLO, C.S.; NERY, J.T. Distribuições de extremos de temperatura mínima no estado do Paraná. Revista Brasileira de Agrometeorologia, v.8, p.247-253, 2000.

SCHICKEDANZ, P.T.; KRAUSE, G.F.A test for the scale parameters of two gamma distributions using the generalized likelihood ratio. Journal of Applied Meteorology, v.9, p.13-16, 1969.

VINCENT, L.A.; PETERSON, T.C.; BARROS, V.R.; MARINO, M.B.; RUSTICUCCI, M.; CARRASCO, G.; RAMIREZ, E.; ALVES, L.M.; AMBRIZZI, T.; BERLATO, M.A.; GRIMM, A.M.; MARENGO, J.A.; MOLION. L.; MONCUNILL, D.F.; REBELLO, E.; ANUNCIAÇÃO, Y.M.T.; QUINTANA, J.; SANTOS, J.L.; BAEZ, J.; CORONEL, G.; GARCIA, J.; TREBEJO, I.; BIDEGAIN, M.; HAYLOCK, M.R.; KAROLY, D. Observed trends in indices of daily temperature extremes in South America 1960-2000. Journal of Climate, v.18, p.50115023, 2005.

VON STORCH, H.; ZWIERS, F.W. Statistical Analysis in Climate Research. 1.ed. Cambridge: Cambridge University Press, 1999. 494p.

WILKS, D,S, Theoretical probability distributions, In: Statistical methods in the atmospheric sciences, 1.ed. San Diego: Academic Press, 1995. 467p.

WILKS, D.S. Theoretical probability distributions. In: Statistical methods in the atmospheric sciences. San Diego: Academic Press, 2006. 592 p. 